\title{
On Free Description Logics with Definite Descriptions
}

\author{
Alessandro Artale ${ }^{1}$, Andrea Mazzullo ${ }^{1}$, Ana Ozaki ${ }^{2}$, Frank Wolter ${ }^{3}$ \\ ${ }^{1}$ Free University of Bozen-Bolzano \\ ${ }^{2}$ University of Bergen \\ ${ }^{3}$ University of Liverpool \\ \{artale,mazzullo\}@inf.unibz.it, ana.ozaki@uib.no,wolter@liverpool.ac.uk
}

\begin{abstract}
Definite descriptions are phrases of the form 'the $x$ such that $\varphi$ ', used to refer to single entities in a context. They are often more meaningful to users than individual names alone, in particular when modelling or querying data over ontologies. We investigate free description logics with both individual names and definite descriptions as terms of the language, while also accounting for their possible lack of denotation. We focus on the extensions of $\mathcal{A L C}$ and, respectively, $\mathcal{E} \mathcal{L}$ with nominals, the universal role, and definite descriptions. We show that standard reasoning in these extensions is not harder than in the original languages, and we characterise the expressive power of concepts relative to first-order formulas using a suitable notion of bisimulation. Moreover, we lay the foundations for automated support for definite descriptions generation by studying the complexity of deciding the existence of definite descriptions for an individual under an ontology. Finally, we provide a polynomial-time reduction of reasoning in other free description logic languages based on dual-domain semantics to the case of partial interpretations.
\end{abstract}

\section{Introduction}

Noun phrases that can be used to refer to a single object in a context are known in linguistics as referring expressions (REs). These include both individual names, such as 'KR 2021', and definite descriptions, such as 'the General Chair of KR 2021' (Neale 1990; Cann 1993). Compared to individual names alone, REs provide increased flexibility in the description and the identification of objects, representing also a natural tool to transmit this kind of information in a semantically transparent way. In the context of information and knowledge base (KB) management systems, REs have been proposed to address the problem of object identifiers that remain obscure to end-users, such as blank node identifiers in RDF or system-generated ref expressions in objectoriented databases (Borgida, Toman, and Weddell 2016b; Borgida, Toman, and Weddell 2017).

However, with the recent exception of the work by Neuhaus, Kutz, and Righetti (2020) discussed below, most of the ontology languages considered in the literature have not included definite descriptions as first-class terms, on a par with individual names. To this goal, another feature of REs has to be taken into account: that of possibly failing to denote any object at all. For instance, 'KR 2019' is a non-denoting individual name, since no KR conference took place in 2019, while 'the Program Chair of KR 2020' and 'the banquet of KR 2020' are nondenoting definite descriptions, because this conference had two Program Chairs and no banquet in 2020. This is not easily captured in classical first-order logic (FO), where an individual name is always assigned to an element of the domain by the interpretation function, and definite descriptions are not included among the terms of the language (Russell 1905). Logics that allow for possibly nondenoting terms are known as free logics (Bencivenga 2002; Lehmann 2002).

In this work, we introduce and study a family of description logic (DL) languages with both individual names and definite descriptions, that we call free DLs with definite descriptions, or free DLs, for short. Syntactically, they extend the classical ones with nominals of the form $\{\iota C\}$, where $\iota C$ is a term standing for the definite description 'the object that is $C^{\prime}$ and $C$ is a concept. We denote the resulting DLs with an upperscript $\iota$, focussing in particular on $\mathcal{A L C O}_{u}^{\iota}$ and $\mathcal{E} \mathcal{L} \mathcal{O}_{u}^{\iota}$, which are, respectively, $\mathcal{A L C}$ and $\mathcal{E} \mathcal{L}$ with nominals, the universal role, and definite descriptions. Their semantics is based on partial interpretations, that generalise the classical ones by letting the interpretation function to be partial on individual names, meaning that only a subset of all the individual names has its elements assigned to objects of the domain. Moreover, the extension of $\{\iota C\}$ in a partial interpretation coincides with that of the concept $C$, if $C$ is interpreted as a singleton, and it is empty otherwise. Nominals involving definite descriptions can be used to form concept inclusions (CIs) with different satisfaction conditions. E.g.,

$$
\{\iota \exists \text { isPCof. }\{\text { dl20 }\} \subseteq \exists \text { reportsTo. }\{\iota \exists \text { isGCof. }\{\text { dl20 }\}\}
$$

states that whoever (if anyone) is the Program Chair of DL 2020 reports to the General Chair of DL 2020: if there is exactly one object in $\exists$ isPCof. $\{\mathrm{dl} 20\}$, then $\exists$ isGCof. $\{\mathrm{dl} 20\}$ is forced to have exactly one element as well, but this $\mathrm{CI}$ is (vacuously) satisfied also in interpretations without, or with more than one, object in $\exists$ isPCof. $\{\mathrm{d} \mid 20\}$.

We show that reasoning in free DLs with definite descriptions can be performed at no additional costs. For (extensions of) $\mathcal{A} \mathcal{L C O}_{u}^{\iota}$, we employ a polynomial time reduction (via a translation that can be applied to other constructors as well) to the corresponding language without definite descriptions, so that efficient off-the-shelf reasoners can be 
used. Moreover, we show that entailment in $\mathcal{E} \mathcal{L} \mathcal{O}_{u}^{\iota}$ ontologies remains tractable, using a modified version of the algorithm for classical $\mathcal{E} \mathcal{L O}$ (Baader, Brandt, and Lutz 2005).

We next characterise the expressive power of $\mathcal{A L C O}_{u}^{\iota}$ concepts relative to $\mathrm{FO}$ on partial interpretations, using an appropriate notion of $\mathcal{A L C O} \mathcal{L}_{u}^{\iota}$ bisimulations. This result is of interest in its own right, but also serves as an important technical tool for the remainder of the article.

Having designed a suitable DL language, we further consider the task of constructing ontologies with definite descriptions. As a step in this direction, we study the problem of finding meaningful REs for an individual under a given ontology. This is related to $R E$ generation in natural language processing, concerned with the automatic production of such noun phrases, possibly extracted from a nonlinguistic source, e.g. a database (Reiter and Dale 2000; Krahmer, van Erk, and Verleg 2003; Krahmer and van Deemter 2012). Towards a better understanding of this problem, we investigate the complexity of deciding the existence of an RE for an individual within a given language and signature and with respect to an ontology. The signature allows the user to specify the features of interest for describing an object, and by deciding this problem it can be determined whether alternative characterisations of an individual are available. For example, consider the following $\mathcal{A L C O} \mathcal{L}_{u}^{\iota}$ ontology $\mathcal{O}$, about KR events held between 2018 and 2020:

$$
\begin{aligned}
& \mathrm{KRConf} \equiv\{\mathrm{kr} 18, \mathrm{kr} 19, \mathrm{kr} 20\} \text {, } \\
& \text { KRWork } \equiv\{\mathrm{dl} 18, \mathrm{dl} 19, \mathrm{dl} 20\} \text {, } \\
& \text { KREvent } \equiv \text { KRConf } \sqcup \text { KRWork, } \\
& \{\mathrm{kr} 19\} \sqsubseteq \perp,\{\mathrm{kr} 18\} \equiv \exists \text { hasRC. } \top \text {, } \\
& \{\mathrm{dl} 18, \mathrm{kr} 18, \mathrm{dl} 19, \mathrm{kr} 20\} \equiv \exists \text { hasPCM. }\{\iota \exists \text { isGCof. }\{\mathrm{dl} 20\}\}, \\
& \{\mathrm{kr} 20, \mathrm{dl} 20\} \equiv \exists \text { hasLoc.VirtualLoc. }
\end{aligned}
$$

The first three CIs define, respectively, the concepts of KR Conference, Workshop and Event, while the next two state, respectively, that 'KR 2019' does not denote, and that KR 2018 is the one and only object that has a Registration Chair. The subsequent CI asserts that the KR events having as PC Member the General Chair of DL 2020 are exactly DL 2018, KR 2018, DL 2019 and KR 2020. Finally, the last one expresses that the objects having a virtual location are exactly KR 2020 and DL 2020. Focussing on location-based characterisations of KR events, the nominal $\{\mathrm{kr} 20\}$ has an $\mathcal{E} \mathcal{L O} \mathrm{RE}$ in terms of the signature $\Sigma_{1}=$ $\{$ KRConf, hasLoc, VirtualLoc \}, since

$$
\mathcal{O} \models\{\mathrm{kr} 20\} \equiv \text { KRConf } \sqcap \exists \text { hasLoc.VirtualLoc, }
$$

whereas there is no RE for $\{\mathrm{kr} 20\}$ in any language if we consider the signature $\Sigma_{2}=\{$ KREvent, hasLoc, VirtualLoc . If we instead choose to refer to KR events in light of their organising members, we have that $\{k r 20\}$ has no RE under $\Sigma_{3}=\{$ KRConf, hasPCM, isGCof, dl20\}, while it can be described in $\mathcal{A L C O}_{u}^{\iota}$ (but not in $\mathcal{E} \mathcal{L} \mathcal{O}_{u}^{\iota}$ ) in terms of $\Sigma_{4}=\{$ KRConf, hasRC, hasPCM, isGCof, dl20 $\}$, since it is equivalent under $\mathcal{O}$ to the $\mathcal{A L C O}_{u}^{\iota}$ concept

$$
\text { KRConf } \sqcap \exists \text { hasPCM. }\{\iota \exists \text { isGCof. }\{d 20\}\} \sqcap \neg \exists \text { hasRC. } T \text {. }
$$

We show that deciding the existence of REs is 2EXPTIME-complete if the ontology and the RE are both in $\mathcal{A L C O}_{u}^{\iota}$. The problem is in PTIME if the ontology and the RE are both in $\mathcal{E} \mathcal{L} \mathcal{O}_{u}^{\iota}$, under the additional assumption that the individual name one aims to describe denotes in every model of the ontology. Without this assumption, the complexity remains open. If FO expressions are allowed as REs, the first problem becomes EXPTIME-complete and the latter is still in PTIME, because of the projective Beth definability property (Beth 1956; Chang and Keisler 1990) of $\mathrm{FO}$, also on partial interpretations, and because reasoning in $\mathcal{A} \mathcal{L} \mathcal{O}_{u}^{\iota}$ and $\mathcal{E} \mathcal{L} \mathcal{O}_{u}^{\iota}$ are EXPTIME- and, respectively, PTIME-complete. In this case, for $\mathcal{E} \mathcal{L} \mathcal{O}_{u}^{\iota}$, no restriction is needed regarding the denotation of the individual name. If instead the ontology is in $\mathcal{A L C O}_{u}^{\iota}$ (even $\mathcal{A L C O}$ ), but one asks for an $\mathcal{E} \mathcal{L} \mathcal{O}_{u}^{\iota} \mathrm{RE}$, the problem becomes undecidable.

Finally, we compare our framework to the positive and negative semantics for free DLs proposed by Neuhaus, Kutz, and Righetti (2020), based on dual-domain interpretations. For both these semantics, we provide a polynomial time reduction to reasoning in $\mathcal{A L C O}_{u}^{\iota}$ on partial interpretations.

Detailed proofs are provided in the full version (Artale et al. 2021a).

\section{Free Description Logics}

We introduce basic notions for free DLs (with definite descriptions) by presenting the syntax and semantics of $\mathcal{A} \mathcal{L C O} \mathcal{O}_{u}^{\iota}$, which we define as a free DL based on the classical $\mathcal{A L C O}_{u}$ (Baader et al. 2003), and other related languages.

\subsection{Syntax}

Let $N_{C}, N_{R}$ and $N_{I}$ be countably infinite and pairwise disjoint sets of concept names, role names, and individual names, respectively. The $\mathcal{A} \mathcal{L C O} \mathcal{O}_{u}^{\iota}$ terms $\tau$ and concepts $C$ are constructed by mutual induction as follows:

$\tau::=a|\iota C, C::=A|\{\tau\}|\neg C|(C \sqcap C)|\exists r . C| \exists u . C$, where $a \in \mathrm{N}_{1}, A \in \mathrm{N}_{\mathrm{C}}, r \in \mathrm{N}_{\mathrm{R}}$, and $u$ is the universal role. A term of the form $\iota C$ is called a definite description, with the concept $C$ being the body of $\iota C$, and a concept $\{\tau\}$ is called a (term) nominal. An $\mathcal{A} \mathcal{L C O} \mathcal{C}_{u}^{\iota}$ axiom is either an $\mathcal{A L C O}_{u}^{\iota}$ concept inclusion $(C I)$ of the form $(C \sqsubseteq D)$ or an $\mathcal{A L C O}_{u}^{\iota}$ assertion of the form $C(\tau)$ or $r\left(\tau_{1}, \tau_{2}\right)$, where $C, D$ are concepts, $r \in \mathrm{N}_{\mathrm{R}}$, and $\tau, \tau_{1}, \tau_{2}$ are terms. An $\mathcal{A L C} \mathcal{L}_{u}^{\iota}$ ontology $\mathcal{O}$ is a finite set of CIs and assertions.

All the usual syntactic abbreviations and conventions are assumed. In particular, for concepts, we set $\perp=A \sqcap \neg A$, $\top=\neg \perp, C \sqcup D=\neg(\neg C \sqcap \neg D), C \Rightarrow D=\neg C \sqcup D$, and $\forall s . C=\neg \exists s . \neg C$, with $s \in \mathrm{N}_{\mathrm{R}} \cup\{u\}$, while a concept equivalence $(C E) C \equiv D$ abbreviates $C \sqsubseteq D, D \sqsubseteq C$.

In the rest of this paper, we will consider other DL languages with nominals, that we introduce briefly here. We define the classical $\mathcal{A L C O}$ as $\mathcal{A L C O} \mathcal{L C}_{u}^{\iota}$ without neither definite descriptions nor the universal role, while $\mathcal{A L C O}{ }^{\iota}$ and $\mathcal{A L C O}_{u}$ are defined as $\mathcal{A L C O}$ with the addition of either definite descriptions or the universal role, respectively. Moreover, the language $\mathcal{E} \mathcal{L O}_{u}^{\iota}$ is obtained from $\mathcal{A} \mathcal{L C O} \mathcal{O}_{u}^{\iota}$ by allowing only for $\perp, \top$ (considered now as primitive logical 
symbols), concept names, term nominals, conjunctions and existential restrictions. Finally, $\mathcal{E} \mathcal{L} \mathcal{O}, \mathcal{E} \mathcal{L} \mathcal{O}^{\iota}$ and $\mathcal{E} \mathcal{L} \mathcal{O}_{u}$ are similarly defined sublanguages of $\mathcal{E} \mathcal{L} \mathcal{O}_{u}^{\iota}$.

Given a DL $\mathcal{L}$, the signature of an $\mathcal{L}$ ontology $\mathcal{O}, \Sigma_{\mathcal{O}}$, is the set of all concept, role and individual names occurring in $\mathcal{O}$, while $\operatorname{con}(\mathcal{O})$ is the set of all subconcepts occurring in $\mathcal{O}$. For a signature $\Sigma \subseteq \mathrm{N}_{\mathrm{C}} \cup \mathrm{N}_{\mathrm{R}} \cup \mathrm{N}_{\mathrm{l}}$, an $\mathcal{L}(\Sigma)$ ontology $\mathcal{O}$ is an $\mathcal{L}$ ontology such that $\Sigma_{\mathcal{O}} \subseteq \Sigma$ (analogous notions are given for $\mathcal{L}$ concepts, where in particular con $(C)$ is the set of subconcepts occurring in $C$ ).

\subsection{Semantics}

For the DL languages with nominals considered in this work, we generalise their semantics through the notion of partial interpretation. A partial interpretation is a pair $\mathcal{I}=$ $\left(\Delta^{\mathcal{I}},{ }^{\mathcal{I}}\right)$, where $\Delta^{\mathcal{I}}$ is a non-empty set, called domain of $\mathcal{I}$, and ${ }^{\mathcal{I}}$ is a function that maps every $A \in \mathrm{N}_{\mathrm{C}}$ to a subset of $\Delta^{\mathcal{I}}$, every $r \in N_{\mathrm{R}}$ to a subset of $\Delta^{\mathcal{I}} \times \Delta^{\mathcal{I}}$, the universal role $u$ to the set $\Delta^{\mathcal{I}} \times \Delta^{\mathcal{I}}$ itself, and every $a$ in a subset of $N_{1}$ to an element in $\Delta^{\mathcal{I}}$. In other words, ${ }^{\mathcal{I}}$ is a total function on $\mathrm{N}_{\mathrm{C}} \cup \mathrm{N}_{\mathrm{R}}$ and a partial function on $\mathrm{N}_{\mathrm{I}}$. A total interpretation is a partial interpretation $\mathcal{I}=\left(\Delta^{\mathcal{I}},{ }^{\mathcal{I}}\right)$ in which $\cdot{ }^{\mathcal{I}}$ is also total on $N_{1}$. The value $\tau^{\mathcal{I}}$ of a term $\tau$ in $\mathcal{I}$ and the extension $C^{\mathcal{I}}$ of a concept $C$ in $\mathcal{I}$ are defined by mutual induction:

$$
(\iota C)^{\mathcal{I}}= \begin{cases}d, & \text { if } C^{\mathcal{I}}=\{d\}, \text { for some } d \in \Delta^{\mathcal{I}} \\ \text { undefined, } & \text { otherwise. }\end{cases}
$$

We say that $\tau$ denotes in $\mathcal{I}$ iff $\tau^{\mathcal{I}}=d$, for a $d \in \Delta^{\mathcal{I}}$. Thus, in particular, an individual name $a$ denotes in $\mathcal{I}$ iff $a^{\mathcal{I}}$ is defined. In addition, where $s \in \mathrm{N}_{\mathrm{R}} \cup\{u\}$ :

$$
\begin{gathered}
(\neg C)^{\mathcal{I}}=\Delta^{\mathcal{I}} \backslash C^{\mathcal{I}}, \quad(C \sqcap D)^{\mathcal{I}}=C^{\mathcal{I}} \cap D^{\mathcal{I}}, \\
(\exists s . C)^{\mathcal{I}}=\left\{d \in \Delta^{\mathcal{I}} \mid \exists e \in C^{\mathcal{I}}:(d, e) \in s^{\mathcal{I}}\right\} .
\end{gathered}
$$

Moreover, we set $\{\tau\}^{\mathcal{I}}=\left\{\tau^{\mathcal{I}}\right\}$, if $\tau$ denotes in $\mathcal{I}$, and $\{\tau\}^{\mathcal{I}}=\emptyset$, otherwise.

A concept $C$ is satisfied in $\mathcal{I}$ iff $C^{\mathcal{I}} \neq \emptyset$, and it is satisfiable iff there is a partial interpretation in which it is satisfied. Given an axiom $\alpha \in \mathcal{O}$, the satisfaction of $\alpha$ in $\mathcal{I}$, written $\mathcal{I} \models \alpha$, is defined as follows:

$$
\begin{gathered}
\mathcal{I} \models C \sqsubseteq D \text { iff } C^{\mathcal{I}} \subseteq D^{\mathcal{I}}, \\
\mathcal{I} \models C(\tau) \text { iff } \tau \text { denotes in } \mathcal{I} \text { and } \tau^{\mathcal{I}} \in C^{\mathcal{I}}, \\
\mathcal{I} \models r\left(\tau_{1}, \tau_{2}\right) \text { iff } \tau_{1}, \tau_{2} \text { denote in } \mathcal{I} \text { and }\left(\tau_{1}^{\mathcal{I}}, \tau_{2}^{\mathcal{I}}\right) \in r^{\mathcal{I}} .
\end{gathered}
$$

We say that $\mathcal{O}$ is satisfied in a partial interpretation $\mathcal{I}$ (or that $\mathcal{I}$ satisfies, or is a model of, $\mathcal{O}$ ), written $\mathcal{I}=\mathcal{O}$, iff $\mathcal{I}=\alpha$, for every $\alpha \in \mathcal{O}$, and it is satisfiable iff it is satisfied in some partial interpretation. A concept $C$ is satisfiable w.r.t. an ontology $\mathcal{O}$ if both $C$ and $\mathcal{O}$ are satisfied in some partial interpretation. Moreover, $\mathcal{O}$ entails an axiom $\alpha$, written $\mathcal{O} \models \alpha$, if every partial interpretation that satisfies $\mathcal{O}$ satisfies also $\alpha$. Finally, we say that an ontology $\mathcal{O}^{\prime}$ is a conservative extension of an ontology $\mathcal{O}$ if every model of $\mathcal{O}^{\prime}$ is a model of $\mathcal{O}$, and every model of $\mathcal{O}$ can be turned into a model of $\mathcal{O}^{\prime}$ by modifying the interpretation of symbols in $\Sigma_{\mathcal{O}^{\prime}} \backslash \Sigma_{\mathcal{O}}$, while keeping fixed the interpretation of symbols in $\Sigma_{\mathcal{O}}$. We also consider these notions for total (that is, classical) interpretations and write 'on total interpretations' explicitly whenever this is the case.

\subsection{Basic Properties}

We discuss some properties of free DLs, where $\mathcal{L} \in$ $\left\{\mathcal{A} \mathcal{L} \mathcal{C O}_{u}^{\iota}, \mathcal{E} \mathcal{L O}_{u}^{\iota}\right\}$ in the following.

(1) An $\mathcal{L}$ term $\tau$ denotes in a partial interpretation $\mathcal{I}$ iff $\mathcal{I} \equiv \top \sqsubseteq \exists u .\{\tau\}$. Furthermore, an $\mathcal{L}$ ontology $\mathcal{O}$ entails $\top \sqsubseteq \exists u .\{\tau\}$ iff $\mathcal{O} \equiv \top(\tau)$ and this happens iff $\tau$ denotes in all the partial interpretations that are models of $\mathcal{O}$. We say that an individual name a denotes w.r.t. an ontology $\mathcal{O}$ if $\mathcal{O} \models \top \sqsubseteq \exists u .\{a\}$. By adding such a CI to an ontology for each individual name occurring in it, we immediately obtain that the $\mathcal{L}$ ontology satisfiability and entailment problems on total interpretations can be reduced in polynomial time to the corresponding problems on partial interpretations. The converse polynomial time reduction can be defined by substituting every individual name $a$ occurring in an ontology or axiom with a fresh concept name $B_{a}$, and adding the CI $B_{a} \sqsubseteq\left\{b_{a}\right\}$, for a fresh individual name $b_{a}$.

(2) On partial interpretations, an $\mathcal{L}$ assertion $C(\tau)$ is not equivalent to $\{\tau\} \sqsubseteq C$. Indeed, while terms occurring in assertions are forced by the semantics to always denote, the CI $\{\tau\} \sqsubseteq C$ is satisfied in any partial interpretation where $\tau$ is not denoting. Nevertheless, assertions are just syntactic sugar. One can replace

- $C(\tau)$ by $\{\tau\} \sqsubseteq C, \top \sqsubseteq \exists u \cdot\{\tau\}$; and

- $r\left(\tau_{1}, \tau_{2}\right)$ by $\left\{\tau_{1}\right\} \sqsubseteq \exists r .\left\{\tau_{2}\right\}, \top \sqsubseteq \exists u .\left\{\tau_{1}\right\}$.

This encoding yields an equivalent ontology. Thus, from now on, we may assume w.l.o.g. that $\mathcal{L}$ ontologies do not contain assertions.

(3) For every $\mathcal{L}$ ontology $\mathcal{O}$, concept $C$ and term $\tau$, we have that $\mathcal{O} \models\{\tau\} \equiv C$ implies $\mathcal{O} \models\{\tau\} \equiv\{\iota C\}$. In $\mathcal{E} \mathcal{L} \mathcal{O}_{u}^{\iota}$, under the assumption that an $\mathcal{E} \mathcal{L} \mathcal{O}_{u}^{\iota}$ term $\tau$ denotes in every model of an $\mathcal{E} \mathcal{L} \mathcal{O}_{u}^{\iota}$ ontology $\mathcal{O}$, we also have the following, for every $\mathcal{E} \mathcal{L} \mathcal{O}_{u}^{\iota}$ concept $C$ : if $\mathcal{O} \models\{\tau\} \equiv C$, then $\mathcal{O} \models\{\tau\} \equiv C^{\prime}$, where $C^{\prime}$ is obtained from $C$ by substituting every $\{\iota D\}$ occurring in $C$ with the concept $D$.

(4) Given an $\mathcal{L}$ ontology $\mathcal{O}$, we can obtain a conservative extension $\overline{\mathcal{O}}$ of $\mathcal{O}$ in flattened form, that is, such that all occurrences of definite descriptions in $\overline{\mathcal{O}}$ are of the form $\iota B$, where $B$ is a concept name. Indeed, let $\iota C_{1}, \ldots, \iota C_{n}$ be all the definite descriptions in $\mathcal{O}$ that do not occur in the body of another definite description $\iota C^{\prime}$. We define $\overline{\mathcal{O}}$ as

$$
\mathcal{O}^{\prime} \cup \bigcup_{1 \leq i \leq n} \overline{\left\{B_{C_{i}} \equiv C_{i}\right\}}
$$

where $\mathcal{O}^{\prime}$ is obtained from $\mathcal{O}$ by substituting the bodies $C_{1}, \ldots, C_{n}$ of $\iota C_{1}, \ldots, \iota C_{n}$ with fresh concept names $B_{C_{1}}, \ldots, B_{C_{n}}$, respectively, and $\overline{\left\{B_{C_{i}} \equiv C_{i}\right\}}$ is the ontology obtained by recursively applying the procedure just described to the ontology $\left\{B_{C_{i}} \equiv C_{i}\right\}$.

\section{Reasoning in Free DLs}

We study the complexity of reasoning in $\mathcal{A} \mathcal{L C O} \mathcal{O}_{u}^{\iota}$ and $\mathcal{E} \mathcal{L} \mathcal{O}_{u}^{\iota}$.

\subsection{Satisfiability in $\mathcal{A L C O} \mathcal{L C}_{u}^{\iota}$}

We prove that satisfiability in $\mathcal{A L C O}_{u}^{\iota}$ is ExPTIMEcomplete. To show this result, we provide a polynomial size equisatisfiable translation into $\mathcal{A L C O}{ }_{u}$. 
An $\mathcal{A L C O}_{u}^{\iota}$ ontology $\mathcal{O}$ is in normal form if it is in flattened form and all the CIs in $\mathcal{O}$ are either of the form $E \sqsubseteq F$, where $E, F$ are $\mathcal{A L C}_{u}$ (i.e., $\mathcal{A L C}$ with the universal role) concepts, or $\{\tau\} \sqsubseteq A$, or $A \sqsubseteq\{\tau\}$, with $A \in \mathrm{N}_{\mathrm{C}}$. It can be seen that an $\mathcal{A} \mathcal{L} \mathcal{C O}_{u}^{\iota}$ ontology can be transformed in polynomial time into an $\mathcal{A L C O}_{u}^{\iota}$ ontology in normal form that is a conservative extension of the original.

We now define a translation of an $\mathcal{A} \mathcal{L C} \mathcal{O}_{u}^{\iota}$ ontology $\mathcal{O}$ in normal form into an $\mathcal{A L C O}_{u}$ ontology $\mathcal{O}^{\dagger}$. While the translation preserves symbols in $\mathrm{N}_{\mathrm{C}} \cup \mathrm{N}_{\mathrm{R}}$, nominals $\{\tau\}$ are translated as follows:

$$
\{\tau\}^{\dagger}=\{\tau\}^{+} \sqcap C_{\tau}^{\leq 1},
$$

where: $\{\tau\}^{+}=A_{b}$, with fresh $A_{b} \in \mathrm{N}_{\mathrm{C}}$, if $\tau=b \in \mathrm{N}_{\mathrm{I}}$; $\{\tau\}^{+}=B$, if $\tau=\iota B$, with $B \in \mathrm{N}_{\mathrm{C}}$; and $C_{\bar{\tau}}^{\leq 1}$ stands for the concept $\forall u .\left(\{\tau\}^{+} \Rightarrow\left\{a_{\tau}\right\}\right)$, with fresh $a_{\tau} \in \mathrm{N}_{\mathrm{I}}$. We now define

$$
\begin{gathered}
(E \sqsubseteq F)^{\dagger}=E \sqsubseteq F, \\
(\{\tau\} \sqsubseteq A)^{\dagger}=\{\tau\}^{\dagger} \sqsubseteq A, \quad(A \sqsubseteq\{\tau\})^{\dagger}=A \sqsubseteq\{\tau\}^{\dagger},
\end{gathered}
$$

where $E, F$ are $\mathcal{A L C}_{u}$ (i.e., $\mathcal{A L C}$ with the universal role) concepts, and $A$ is a concept name. Finally, we set $\mathcal{O}^{\dagger}$ as

$$
\bigcup_{C \sqsubseteq D \in \mathcal{O}}\left\{(C \sqsubseteq D)^{\dagger}\right\} \underset{\{\tau\} \in \operatorname{con}(\mathcal{O})}{\cup}\left\{\{\tau\}^{+} \sqsubseteq \forall u \cdot\left(\left\{a_{\tau}\right\} \Rightarrow\{\tau\}^{+}\right)\right\} .
$$

We then obtain the following.

Lemma 1. An $\mathcal{A L C O}_{u}^{\iota}$ ontology $\mathcal{O}$ in normal form is satisfiable iff the $\mathcal{A L C O}_{u}$ ontology $\mathcal{O}^{\dagger}$ is satisfiable on total interpretations.

It follows from a result in Propositional Dynamic Logic extended with nominals and the universal modality (Passy and Tinchev 1991, Corollary 7.7) that the $\mathcal{A L C O}_{u}$ ontology satisfiability problem on total interpretations is in EXPTIME. The matching lower bound comes from the $\mathcal{A L C}$ ontology satisfiability problem on total interpretations (Gabbay et al. 2003). Since the $\mathcal{A} \mathcal{L C} \mathcal{O}_{u}^{\iota}$ ontology satisfiability problem on total interpretations is reducible in polynomial time to its counterpart on partial interpretations (cf. Point (1) in Section 2.3), the following holds.

Theorem 2. $\mathcal{A} \mathcal{L C O} \mathcal{O}_{u}^{\iota}$ ontology satisfiability (both on partial and total interpretations) is EXPTIME-complete.

The reduction we presented can be easily adapted to deal with more expressive DLs, e.g. extensions of $\mathcal{A L C O}_{u}^{\iota}$ with inverse roles and number restrictions.

\subsection{Reasoning in $\mathcal{E} \mathcal{L} \mathcal{O}_{u}^{\iota}$}

We prove that entailment in $\mathcal{E} \mathcal{L} \mathcal{O}_{u}^{\iota}$ ontologies is PTIMEcomplete. To show this result, we assume w.l.o.g. that the assertions are encoded within the CIs in the ontology (cf. Point (2) in Section 2.3) and adapt the completion algorithm for $\mathcal{E} \mathcal{L O}$ ontologies (Baader, Brandt, and Lutz 2005). The main idea is to add a copy of each concept name in an ontology and remove it only if its extension is a singleton in any model. Even though $\mathcal{E} \mathcal{L} \mathcal{O}_{u}^{\iota}$ admits a mild form of disjunction $(\{\iota A\} \sqsubseteq B$ states that the extension of $A$ contains at least two elements or $A \sqsubseteq B$ ), the logic remains 'Horn' in the sense that (if an ontology is satisfiable, then) minimal models exist.

Any $\mathcal{E} \mathcal{L} \mathcal{O}_{u}^{\iota}$ ontology can be converted in polynomial time into a conservative extension in normal form, that is, an $\mathcal{E} \mathcal{L O}_{u}^{\iota}$ ontology $\mathcal{O}$ in flattened form where all CIs have one of the following forms:

$C_{1} \sqcap C_{2} \sqsubseteq D, \exists r . C \sqsubseteq D, C \sqsubseteq \exists r . D,\{\tau\} \sqsubseteq D, C \sqsubseteq\{\tau\}$, where $C_{(i)} \in \mathrm{N}_{\mathrm{C}} \cup\{\top\}, D \in \mathrm{N}_{\mathrm{C}} \cup\{\top, \perp\}$ and all terms $\tau$ in $\mathcal{O}$ are either of the form $\{a\}$, with $a \in \mathrm{N}_{\mathrm{l}}$, or of the form $\{\iota A\}$, with $A \in \mathrm{N}_{\mathrm{C}}$.

Let $\mathcal{O}$ be in normal form. We denote by $\mathcal{B C}_{\mathcal{O}}$ the union of $\{T\}$, the set of all concept names occurring in $\mathcal{O}$, and the set of all concepts $\{a\} \in \operatorname{con}(\mathcal{O})$. Also, we denote by $\mathcal{B C}_{\mathcal{O}}^{+}$ the union of $\mathcal{B C}_{\mathcal{O}}$ with $\{\perp\} \cup\{\{\iota A\} \mid\{\iota A\} \in \operatorname{con}(\mathcal{O})\}$ and by $\mathcal{R}_{\mathcal{O}}$ the set including $u$ and the role names occurring in $\mathcal{O}$. Given $A, B \in \mathrm{N}_{\mathrm{C}}$, we may write $A \sqsubseteq B$ instead of $A \sqcap A \sqsubseteq B$. If $\{\iota A\} \in \operatorname{con}(\mathcal{O})$, we assume w.l.o.g. that $\{\iota A\} \sqsubseteq A \in \mathcal{O}$. Moreover, we write $\mathrm{A}$ to denote a concept name which we aim at checking whether $\mathcal{O} \models \mathrm{A} \sqsubseteq B$ (see Lemma 3 and Table 1). The classification graph for $\mathcal{O}$ and A is a tuple $(V, S, R)$ where

- $V=\mathcal{B C}_{\mathcal{O}} \cup\left\{A^{c} \mid A \in\left(\mathcal{B C}_{\mathcal{O}} \cap \mathrm{N}_{\mathrm{C}}\right)\right\}$, with each $A^{c} \in \mathrm{N}_{\mathrm{C}}$ fresh;

- $S$ is a function mapping nodes in $V$ to subsets of $\mathcal{B C}_{\mathcal{O}}^{+}$;

- $R$ is a function mapping edges in $V \times V$ to (possibly empty) subsets of $\mathcal{R}_{\mathcal{O}}$, where $r$ is in $\mathcal{R}_{\mathcal{O}}$.

Intuitively, a concept name of the form $A^{c}$ represents a second element in the extension of $A$, and it is removed from the classification graph if $A$ has at most one object in its extension. Initially, we set $S(C):=\{C, \top\}$, for all nodes $C \in V$, and $R(C, D):=\emptyset$, for all edges $(C, D) \in(V \times V)$. If $C \in V \backslash \mathcal{B C}_{\mathcal{O}}$ is of the form $A^{c}$, with $A \in \mathrm{N}_{\mathrm{C}}$, then we add $A$ to $S\left(A^{c}\right)$. Given $C, D \in \mathcal{B C}_{\mathcal{O}}$, we write $C \sim_{R} D$ iff there are $C_{1}, \ldots, C_{k} \in \mathcal{B C}_{\mathcal{O}}$ such that $C_{1} \in S(C)$; $r \in R\left(C_{j}, C_{j+1}\right)$, for some $r \in \mathcal{R}_{\mathcal{O}}$, for all $1 \leq j<k$; $D \in S\left(C_{k}\right)$. The completion rules are given in Table 1 . Assume that rules are only applied if $S$ or $R$ or $V$ change after the rule application. This bounds the number of rule applications to a polynomial in the number of concept and role names in $\mathcal{O}$. Thus, the resulting completed classification graph for $\mathcal{O}$ can be constructed in polynomial time with respect to the size of $\mathcal{O}$.

Lemma 3. Given an $\mathcal{E} \mathcal{L O}_{u}^{\iota}$ ontology $\mathcal{O}$ in normal form, let $S$ be the node function of a completed classification graph for $\mathcal{O}$ (cf. rules in Table 1), $\mathrm{A} \in \mathrm{N}_{\mathrm{C}}$ and $B \in \mathcal{B C}_{\mathcal{O}} \cup\{\perp\}$. Then, $\mathcal{O}=\mathrm{A} \sqsubseteq B$ iff $S(\mathrm{~A}) \cap\{B, \perp\} \neq \emptyset$.

Thanks to Lemma 3, given arbitrary $\mathcal{E} \mathcal{L} \mathcal{O}_{u}^{\iota}$ concepts $C, D$ and an $\mathcal{E} \mathcal{L} \mathcal{O}_{u}^{\iota}$ ontology $\mathcal{O}$, one can decide in polynomial time whether $C \sqsubseteq D$ is entailed by $\mathcal{O}$ by adding $\mathrm{A} \equiv C$ and $B \equiv D$ to $\mathcal{\mathcal { O }}$, converting it in normal form, and then checking whether $S(\mathrm{~A}) \cap\{B, \perp\} \neq \emptyset$, where A, $B$ are fresh concept names. As an immediate consequence of Lemma 3 and the polynomial size of a completed classification graph we obtain the following complexity result.

Theorem 4. Entailment in $\mathcal{E} \mathcal{L} \mathcal{O}_{u}^{\iota}$ (both on partial and total interpretations) is PTIME-complete. 


\begin{tabular}{|c|c|}
\hline if & then \\
\hline $\mathrm{R}_{1}: C \sqcap D \sqsubseteq B \in \mathcal{O}, C, D \in S(E)$ & add $B$ to $S(E)$ \\
\hline $\mathrm{R}_{2}: \mathrm{A} \sim_{R} E, C \sqsubseteq \exists r . D \in \mathcal{O}, C \in S(E)$ & add $r$ to $R(E, D), R\left(E^{c}, D\right)$ \\
\hline $\mathrm{R}_{3}: \exists r . C \sqsubseteq D \in \overline{\mathcal{O}}, C \in S(B), r \in R(E, B)$ & add $D$ to $S(E)$ \\
\hline $\mathrm{R}_{3}^{\prime}: \exists u . C \sqsubseteq D \in \mathcal{O}, \mathrm{A} \sim_{R} C, E \in V$ & add $D$ to $S(E)$ \\
\hline $\mathrm{R}_{4}:\{\tau\} \in \bar{S}(E) \cap S(D), \mathrm{A} \sim_{R} D$ & $S(E):=S(E) \cup S(D)$ \\
\hline $\mathrm{R}_{5}: r \in R(E, D), \perp \in S(D)$ & add $\perp$ to $S(E)$ \\
\hline $\mathrm{R}_{6}:\{\tau\} \sqsubseteq D \in \mathcal{O},\{\tau\} \in S^{\prime}(E)$ & add $D$ to $S(E)$ \\
\hline $\mathrm{R}_{7}: C \sqsubseteq\{\tau\} \in \mathcal{O}, C \in S(E)$ & add $\{\tau\}$ to $S(E)$ \\
\hline $\mathrm{R}_{8}:\{\tau\} \in S(B), B \in \mathrm{N}_{\mathrm{C}}$ & $V:=V \backslash\left\{B^{c}\right\}$ \\
\hline $\mathrm{R}_{9}: B \in S(E), B^{c} \notin V$ & add $\{\iota B\}$ to $S(E)$ \\
\hline $\mathrm{R}_{10}: \mathrm{A} \sim \sim_{R} C,\{\iota B\} \in S(C)$ & $V:=V \backslash\left\{B^{c}\right\}$ \\
\hline
\end{tabular}

Table 1: Completion rules for subsumption in $\mathcal{E} \mathcal{L} \mathcal{O}_{u}^{\iota}$ with respect to ontologies.

The completed classification graph can be used to define a polynomial size canonical model for an $\mathcal{E} \mathcal{L} \mathcal{O}_{u}^{\iota}$ ontology (if it is satisfiable). Let $\mathcal{O}$ be in normal form and let $(V, S, R)$ be the completed classification graph for $\mathcal{O}$ and a concept name $A$. Consider the following sets:

$$
\begin{aligned}
& \mathcal{R}_{\mathrm{A}}=\left\{C \in \mathcal{B C}_{\mathcal{O}} \mid \mathrm{A} \sim_{R} C\right\}, \\
& \mathcal{R}_{\mathrm{A}}^{c}=\left\{C^{c} \in V \mid C \in \mathcal{R}_{\mathrm{A}} \cap \mathrm{N}_{\mathrm{C}}\right\},
\end{aligned}
$$

over which we define the relation $\sim$, where

$$
C \sim D \text { iff } C=D \text { or }\{\tau\} \in S(C) \cap S(D) \text {, for some } \tau .
$$

It can be seen that $\sim$ is an equivalence relation, whose equivalence classes are denoted by $[C]$. Assume $\perp \notin S(\mathrm{~A})$ (otherwise, by Lemma 3, no model of $\mathcal{O}$ and $A$ exists). The polynomial size canonical model of $\mathcal{O}$ and $\mathrm{A}$ is the partial interpretation $\mathcal{I}_{\mathrm{A}, \mathcal{O}}=\left(\Delta^{\mathcal{I}_{\mathrm{A}, \mathcal{O}}}, \mathcal{I}_{\mathrm{A}, \mathcal{O}}\right)$ such that:

- $\Delta^{\mathcal{I}_{\mathrm{A}, \mathcal{O}}}=\left\{[C] \mid C \in \mathcal{R}_{\mathrm{A}} \cup \mathcal{R}_{\mathrm{A}}^{c}\right\}$

- $r^{\mathcal{I}_{\mathrm{A}, \mathcal{O}}}=\left\{([C],[D]) \in \Delta^{\mathcal{I}_{\mathrm{A}, \mathcal{O}}} \times \Delta^{\mathcal{I}_{\mathrm{A}, \mathcal{O}}} \mid \exists D^{\prime} \in[D] . r \in\right.$ $\left.R\left(C, D^{\prime}\right)\right\}$, for all $r \in \mathrm{N}_{\mathrm{R}} \cap \mathcal{R}_{\mathcal{O}}$;

- $D^{\mathcal{I}_{\mathrm{A}, \mathcal{O}}}=\left\{[C] \in \Delta^{\mathcal{I}_{\mathrm{A}, \mathcal{O}}} \mid D \in S(C)\right\}$, for all $D \in \mathrm{N}_{\mathrm{C}}$;

- $a^{\mathcal{I}_{\mathrm{A}, \mathcal{O}}}=[C]$, for some $C \in \mathcal{R}_{\mathrm{A}}$, if $\{a\} \in S(C)$, for all $a \in \mathrm{N}_{\mathrm{I}}$.

We are now ready to state the main property of the canonical model, used in Section 5.

Theorem 5. Let $\mathcal{O}$ be an $\mathcal{E} \mathcal{L O}_{u}^{\iota}$ ontology in normal form and $\mathrm{A}$ a concept name satisfiable w.r.t. $\mathcal{O}$. Then $[\mathrm{A}] \in C^{\mathcal{I}_{\mathrm{A}, \mathcal{O}}}$ iff $\mathcal{O} \models \mathrm{A} \sqsubseteq C$, for every $\mathcal{E} \mathcal{L} \mathcal{O}_{u}$ concept $C$.

We note that the equivalence is stated for $\mathcal{E} \mathcal{L} \mathcal{O}_{u}$ concepts and not for $\mathcal{E} \mathcal{L} \mathcal{O}_{u}^{\iota}$ concepts. In fact, it is an interesting open problem whether polynomial size canonical models exist that satisfy the equivalence for $\mathcal{E} \mathcal{L} \mathcal{O}_{u}^{\iota}$ concepts.

\section{Bisimulations and Expressive Power}

Here we discuss the expressive power of free DLs. In particular, we define a notion of bisimulation for $\mathcal{A L C O}_{u}^{\iota}$ that we use to characterise the expressive power of concepts relative to FO formulas interpreted on partial interpretations. The definitions are standard in the literature (Areces, Blackburn, and Marx 2001; ten Cate 2005;
Lutz, Piro, and Wolter 2011), but have to be adapted to partial interpretations and definite descriptions.

Let $\mathcal{I}$ and $\mathcal{J}$ be partial interpretations, and let $\Sigma$ be a signature. An $\mathcal{A L C O}(\Sigma)$ bisimulation between $\mathcal{I}$ and $\mathcal{J}$ is a relation $Z \subseteq \Delta^{\mathcal{I}} \times \Delta^{\mathcal{J}}$ such that, for every $d \in \Delta^{\mathcal{I}}$ and $e \in \Delta^{\mathcal{J}}$ with $(d, e) \in Z$, every concept name or nominal $X$ formulated within $\Sigma$, and every role name $r$ in $\Sigma$ : (atom) $d \in X^{\mathcal{I}}$ iff $e \in X^{\mathcal{J}}$; (forth) if $\left(d, d^{\prime}\right) \in r^{\mathcal{I}}$ then there is $e^{\prime} \in \Delta^{\mathcal{J}}$ such that $\left(e, e^{\prime}\right) \in r^{\mathcal{J}}$ and $\left(d^{\prime}, e^{\prime}\right) \in Z$; and (back) if $\left(e, e^{\prime}\right) \in r^{\mathcal{J}}$ then there is $d^{\prime} \in \Delta^{\mathcal{I}}$ such that $\left(d, d^{\prime}\right) \in r^{\mathcal{I}}$ and $\left(d^{\prime}, e^{\prime}\right) \in Z$. For pointed partial interpretations $(\mathcal{I}, d)$ and $(\mathcal{J}, e)$, we say that $(\mathcal{I}, d)$ is $\mathcal{A L C O}(\Sigma)$ bisimilar to $(\mathcal{J}, e)$ and write $(\mathcal{I}, d) \sim_{\Sigma}^{\mathcal{A L C O}}(\mathcal{J}, e)$ if there is an $\mathcal{A L C O}(\Sigma)$ bisimulation $Z$ between $\mathcal{I}$ and $\mathcal{J}$ such that $(d, e) \in Z . \mathcal{A L C O}(\Sigma)$ bisimulations characterise the expressive power of $\mathcal{A} \mathcal{L C O}(\Sigma)$ concepts in the sense that an FO formula $\varphi$ is preserved under $\mathcal{A L C O}(\Sigma)$ bisimulations iff it is equivalent to an $\mathcal{A L C O}(\Sigma)$ concept. To characterise $\mathcal{A L C O}_{u}^{\iota}(\Sigma)$ we add a condition that reflects its ability to count up to one and also add totality conditions that reflect the addition of the universal role.

An $\mathcal{A L C O}_{u}^{\iota}(\Sigma)$ bisimulation $Z$ between $\mathcal{I}$ and $\mathcal{J}$ is an $\mathcal{A L C O}(\Sigma)$ bisimulation that is total, meaning that $\Delta^{\mathcal{I}}$ and $\Delta^{\mathcal{J}}$ are the domain and range of the relation, and that satisfies, for all $(d, e) \in Z$ :

(८) there exists $d^{\prime} \in \Delta^{\mathcal{I}}$ such that $d \neq d^{\prime}$ and $(\mathcal{I}, d) \sim_{\Sigma}^{\mathcal{A} \mathcal{L C O}}\left(\mathcal{I}, d^{\prime}\right)$ iff there exists $e^{\prime} \in \Delta^{\mathcal{J}}$ such that $e^{\prime} \neq e$ and $(\mathcal{J}, e) \sim \sim_{\Sigma}^{\mathcal{A L C O}}\left(\mathcal{J}, e^{\prime}\right)$.

We write $(\mathcal{I}, d) \quad \sim_{\Sigma}^{\mathcal{A} \mathcal{L C} \mathcal{O}_{u}^{\iota}}(\mathcal{J}, e)$ if there exists an $\mathcal{A L C O}_{u}^{\iota}(\Sigma)$ bisimulation $Z$ between $\mathcal{I}$ and $\mathcal{J}$ containing $(d, e)$, and we write $(\mathcal{I}, d) \equiv_{\Sigma}^{\mathcal{A} \mathcal{L C} \mathcal{O}_{u}^{\iota}}(\mathcal{J}, e)$ if $d \in C^{\mathcal{I}}$ iff $e \in$ $C^{\mathcal{J}}$, for all $\mathcal{A L C O}_{u}^{\iota}(\Sigma)$ concepts $C$. The definition of $\omega$ saturated partial interpretation is the obvious generalisation of that one for total interpretations (Chang and Keisler 1990; Artale et al. 2021a).

Theorem 6. For all signatures $\Sigma$ and all pointed partial interpretations $(\mathcal{I}, d)$ and $(\mathcal{J}, e)$,

1. if $(\mathcal{I}, d) \sim_{\Sigma}^{\mathcal{A} \mathcal{L C O} \mathrm{O}_{u}^{\iota}}(\mathcal{J}, e)$, then $(\mathcal{I}, d) \equiv \sum_{\Sigma}^{\mathcal{A} \mathcal{L C O}{ }_{u}^{\iota}}(\mathcal{J}, e)$;

2. if $(\mathcal{I}, d) \equiv \mathcal{A L C O}_{\mathcal{L}}{ }^{\iota}(\mathcal{J}, e)$ and $\mathcal{I}, \mathcal{J}$ are $\omega$-saturated, then $(\mathcal{I}, d) \sim \sim_{\Sigma}^{\mathcal{A} L \mathcal{L} \mathcal{O}^{i}}(\mathcal{J}, e)$ 
The following example illustrates how bisimulations can be used to prove the inexpressibility of certain concepts.

Example 1. The concept $C=\exists u .\{\iota A\}$ states that the extension of $A$ has cardinality one, that is $d \in C^{\mathcal{I}}$ iff $\left|A^{\mathcal{I}}\right|=1$, for any interpretation $\mathcal{I}$ and $d \in \Delta^{\mathcal{I}}$. The concept $D=$ $\exists u .(A \sqcap \neg\{\iota A\})$ is such that $d \in D^{\mathcal{I}}$ iff $\left|A^{\mathcal{I}}\right| \geq 2$, hence it states that $A$ has cardinality greater or equal to two. However, there is no $\mathcal{A L C O}_{u}^{\iota}$ concept stating that the extension of $A$ has cardinality two: the pointed interpretations $(\mathcal{I}, d)$ and $(\mathcal{J}, e)$ depicted below are $\mathcal{A} \mathcal{L C O}_{u}^{\iota}(\{A\})$-bisimilar (witnessed by $\left.Z=\Delta^{\mathcal{I}} \times \Delta^{\mathcal{J}}\right)$, but $\left|A^{\mathcal{I}}\right|=2<\left|A^{\mathcal{J}}\right|$.

$$
{ }^{\mathcal{I}} \cdot A \quad \cdot A \mid e \cdot A \quad \cdot A \quad A^{\mathcal{J}}
$$

We next state that $\mathcal{A} \mathcal{L C O}_{u}^{\iota}$ is the fragment of $\mathrm{FO}$ on partial interpretations that is invariant under $\mathcal{A L C O}_{u}^{\iota}$-bisimulations. The standard translation of an $\mathcal{A L C O}_{u}^{\iota}$ concept $C$ into an FO formula $\pi_{x}(C)$ with one free variable $x$ is defined as expected, where for nominals of the form $\{\iota C\}$ we set:

$$
\begin{aligned}
\pi_{x}(\{\iota C\})= & \exists x \pi_{x}(C) \wedge \forall x \forall y\left(\pi_{x}(C) \wedge \pi_{y}(C) \rightarrow x=y\right) \\
& \wedge \forall y\left(\pi_{y}(C) \rightarrow x=y\right) .
\end{aligned}
$$

An FO formula $\varphi(x)$ is invariant under $\sim \mathcal{A L C O}_{u}^{\iota}$ iff, for every $(\mathcal{I}, d)$ and $(\mathcal{J}, e)$ such that $(\mathcal{I}, d) \sim \sim_{\Sigma}^{\mathcal{A} \mathcal{L C} \mathcal{O}_{u}^{\iota}}(\mathcal{J}, e)$, we have $\mathcal{I}=\varphi(d)$ iff $\mathcal{J}=\varphi(e)$.

Theorem 7. Let $\Sigma$ be a signature, and let $\varphi(x)$ a first-order formula such that $\Sigma_{\varphi(x)} \subseteq \Sigma$. The following conditions are equivalent:

1. there exists an $\mathcal{A L C O}_{u}^{\iota}(\Sigma)$ concept $C$ such that $\pi_{x}(C)$ is logically equivalent to $\varphi(x)$;

2. $\varphi(x)$ is invariant under $\sim_{\Sigma}^{\mathcal{A} \mathcal{L C O} \mathcal{O}_{u}^{\iota}}$.

We next consider $\mathcal{E} \mathcal{L} \mathcal{O}_{u}^{\iota}$. In contrast to $\mathcal{A} \mathcal{L C O} \mathcal{O}_{u}^{\iota}$, we do not have a model-characterisation of $\mathcal{E} \mathcal{L} \mathcal{O}_{u}^{\iota}$ that generalises the one for $\mathcal{E} \mathcal{L}$ (Lutz and Wolter 2010; Lutz, Piro, and Wolter 2011). The fundamental problem is to constrain simulations (the basic notion used to characterise $\mathcal{E} \mathcal{L}$ ) in such a way that they capture the expressivity of $\mathcal{E} \mathcal{L} \mathcal{O}_{u}^{\iota}$ concepts.

To obtain preliminary results on $\mathcal{E} \mathcal{L} \mathcal{O}_{u}^{\iota}$ REs in the next section, we remind the reader of the standard simulations between interpretations and how they characterise $\mathcal{E} \mathcal{L} \mathcal{O}_{u}$. A relation $Z \subseteq \Delta^{\mathcal{I}} \times \Delta^{\mathcal{J}}$ is an $\mathcal{E} \mathcal{L} \mathcal{O}(\Sigma)$ simulation from $\mathcal{I}$ to $\mathcal{J}$ iff it satisfies $\left(\right.$ atom $\left._{R}\right)$, i.e., the 'only if' direction of the Condition (atom), and the Condition (forth) given above. An $\mathcal{E} \mathcal{L O}_{u}(\Sigma)$ simulation from $\mathcal{I}$ to $\mathcal{J}$ is an $\mathcal{E} \mathcal{L O}(\Sigma)$ simulation from $\mathcal{I}$ to $\mathcal{J}$ that is left total, meaning that $\Delta^{\mathcal{I}}$ is the domain of the relation. We write $(\mathcal{I}, d) \leq_{\Sigma} \mathcal{E} \mathcal{L} \mathcal{O}_{u}(\mathcal{J}, e)$ if there exists an $\mathcal{E} \mathcal{L O}_{u}(\Sigma)$ simulation $Z$ from $\mathcal{I}$ to $\mathcal{J}$ with $(d, e) \in Z$. Given a DL $\mathcal{L}$, a partial interpretation $\mathcal{I}$ with $d \in \Delta^{\mathcal{I}}$, and a signature $\Sigma$, we call the $\mathcal{L}(\Sigma)$ type of $d$ in $\mathcal{I}$ the set $t_{\mathcal{L}(\Sigma)}^{\mathcal{I}}(d)$ of $\mathcal{L}(\Sigma)$ concepts $C$ such that $d \in C^{\mathcal{I}}$.

Theorem 8. For all signatures $\Sigma$ and all partial pointed interpretations $(\mathcal{I}, d)$ and $(\mathcal{J}, e)$,

1. if $(\mathcal{I}, d) \leq_{\Sigma}^{\mathcal{E} \mathcal{L O}_{u}}(\mathcal{J}, e)$, then $t_{\mathcal{E} \mathcal{L} \mathcal{O}_{u}(\Sigma)}^{\mathcal{I}}(d) \subseteq t_{\mathcal{E} \mathcal{L} \mathcal{O}_{u}(\Sigma)}^{\mathcal{J}}(e)$;

2. if $t_{\mathcal{E} \mathcal{L} \mathcal{O}_{u}(\Sigma)}^{\mathcal{I}}(d) \subseteq t_{\mathcal{E} \mathcal{L} \mathcal{O}_{u}(\Sigma)}^{\mathcal{J}}(e)$ and $\mathcal{J}$ is $\omega$-saturated, then $(\mathcal{I}, d) \leq_{\Sigma}^{\mathcal{E}} \mathcal{L} \mathcal{O}_{u}(\mathcal{J}, e)$.

\section{Existence of Referring Expressions}

One of the main motivations for enriching DLs with definite descriptions comes from the observation that individual names used in databases, ontologies, or other forms of $\mathrm{KBs}$, are very often completely meaningless to the human user (Borgida, Toman, and Weddell 2016b). Introducing semantically meaningful referring expressions (REs) in addition to individual names via ontologies enables a more informative naming of individuals, and thus a more user-friendly modelling of domains. In this section, we address the problem of providing support for the generation of such expressions for individual names that occur in an ontology. Thus, for an individual name $a$ and an ontology $\mathcal{O}$, the goal is to support the generation of a concept $C$ so that

$$
\mathcal{O} \models\{a\} \equiv C,
$$

if such a concept exists. One can then replace $a$ by $\iota C$ in the ontology or add an explicit definition of $\{a\}$ to the ontology and possibly remove other inclusions that become redundant (see (ten Cate et al. 2006; ten Cate, Franconi, and Seylan 2013) for this approach applied to concept names rather than individual names). Other than being used to improve an ontology, REs may also be regarded as answer to queries about individuals (Borgida, Toman, and Weddell 2016b).

To support the targeted generation of REs, we consider two types of restrictions on $C:(i)$ the restriction of the signature of $C$ to some subset $\Sigma$ of the signature of $\mathcal{O}$; and $(i i)$ restrictions on the DL constructors used in $C$. As an initial step, we focus on the DLs introduced in this paper and on the complexity of deciding the existence of an RE. We also discuss briefly what happens if one admits FO formulas as REs. The algorithm deciding the existence of REs can then inform the development of a generating algorithm for REs, although this is beyond the scope of the present contribution.

Formally, given a pair $\left(\mathcal{L}, \mathcal{L}_{R}\right)$ of logics, $\left(\mathcal{L}, \mathcal{L}_{R}\right) R E$ existence is the problem of deciding, for an $\mathcal{L}$ ontology $\mathcal{O}$, an individual name $a$, and a signature $\Sigma$, whether $a$ is explicitly $\mathcal{L}_{R}(\Sigma)$ definable under $\mathcal{O}$, that is, whether there exists an $\mathcal{L}_{R}(\Sigma)$ concept $C$ such that $\mathcal{O} \models\{a\} \equiv C$. If $\mathcal{L}_{R}=$ FO, then we ask whether there is an FO formula $\varphi(x)$ over $\Sigma$ such that $\mathcal{O} \models \forall x((x=a) \leftrightarrow \varphi(x))$. Such a concept or formula is called an $\mathcal{L}_{R}(\Sigma) R E$ for a under $\mathcal{O}$. If $\mathcal{L}=\mathcal{L}_{R}$, then we simply speak of $\mathcal{L} R E$ existence.

Theorem 9. On partial and total interpretations:

\section{1. $\left(\mathcal{A L C O}_{u}^{\iota}, F O\right)$ RE existence is EXPTIME-complete;}

2. $\left(\mathcal{E} \mathcal{L} \mathcal{O}_{u}^{\iota}, F O\right) R E$ existence is in PTIME;

3. $\mathcal{A L C O}_{u}^{\iota} R E$ existence is 2EXPTIME-complete;

4. $\left(\mathcal{A L C O}_{u}^{\iota}, \mathcal{E} \mathcal{L} \mathcal{O}_{u}^{\iota}\right) R E$ existence is undecidable;

5. $\mathcal{E} \mathcal{L} \mathcal{O}_{u}^{\iota}$ RE existence is in PTIME, for individuals a that denote w.r.t. the ontology.

We first comment on Points (1) and (2), which are consequences of the fact that FO has the projective Beth definability property on total and partial interpretations, and the EXPTIME and PTIME upper bounds for reasoning in $\mathcal{A L C O} \mathcal{L}_{u}^{\iota}$ and $\mathcal{E} \mathcal{L} \mathcal{O}_{u}^{\iota}$, respectively. In detail, we say that an individual name $a$ is implicitly definable from a signature 
$\Sigma$ under an ontology $\mathcal{O}$ if for all (partial or total, respectively) models $\mathcal{I}$ and $\mathcal{J}$ of $\mathcal{O}$ such that $\Delta^{\mathcal{I}}=\Delta^{\mathcal{J}}$ and $X^{\mathcal{I}}=X^{\mathcal{J}}$, for all $X \in \Sigma$, we have $a^{\mathcal{I}}=a^{\mathcal{J}}$. Clearly, if $a$ is explicitly $\mathcal{L}(\Sigma)$-definable under $\mathcal{O}$, then it is implicitly definable from $\Sigma$ under $\mathcal{O}$. We say that $\mathcal{L}$ has the projective Beth definability property $(P B D P)$ for individuals if the converse holds. FO is known to have the PBDP for individuals (and, in fact, for arbitrary relations) under total interpretations (Chang and Keisler 1990). It is straightforward to extend the model-theoretic proof given, e.g., in (Chang and Keisler 1990) to show that FO enjoys the PBDP for individuals (and, again, arbitrary relations) under partial interpretations as well. From an algorithmic viewpoint, the PBDP is important because it implies that explicit definability can be checked using implicit definability, and the latter reduces to a standard reasoning problem: $a$ is implicitly definable from $\Sigma$ under $\mathcal{O}$ iff $\mathcal{O} \cup \mathcal{O}^{\prime}=\{a\} \equiv\left\{a^{\prime}\right\}$, where $\mathcal{O}^{\prime}$ is obtained from $\mathcal{O}$ by uniformly substituting every symbol $X$ not in $\Sigma$ by a fresh symbol $X^{\prime}$. We thus proved Points (1) and (2).

FO is arguably too expressive as a language for REs for individuals in DL ontologies, and Points (3) to (5) address the problem of finding REs within the DLs considered in this paper. Unfortunately, $\mathcal{A} \mathcal{L C} \mathcal{O}_{u}^{\iota}$ does not enjoy the PBDP for individuals.

Example 2. Let $\Sigma=\{A, r\}$ and assume that $\mathcal{O}$ consists of the following CIs:

$$
\begin{gathered}
\{a\} \sqsubseteq \exists r .\{a\}, \\
\neg\{a\} \sqcap A \sqsubseteq \forall r .(\neg\{a\} \Rightarrow \neg A), \\
\neg\{a\} \sqcap \neg A \sqsubseteq \forall r .(\neg\{a\} \Rightarrow A) .
\end{gathered}
$$

Then a is implicitly definable from $\Sigma$ under $\mathcal{O}$ since $\mathcal{O}=$ $\forall x((x=a) \leftrightarrow r(x, x))$. However, the figure depicted below (for which we assume $a^{\mathcal{I}}=$ a) shows a model $\mathcal{I}$ of $\mathcal{O}$ such that $\left(\mathcal{I}, a^{\mathcal{I}}\right) \sim \sim_{\Sigma}^{\mathcal{A L C O} \mathcal{O}_{u}^{\iota}}(\mathcal{I}, e)$, with $e \neq a^{\mathcal{I}}$. Thus, any $\mathcal{A L C O}_{u}^{\iota}(\Sigma)$ concept that applies to $a^{\mathcal{I}}$ applies to e in $\mathcal{I}$ and so a cannot be explicitly $\mathcal{A} \mathcal{L C O} \mathcal{C}_{u}^{\iota}(\Sigma)$ definable under $\mathcal{O}$.

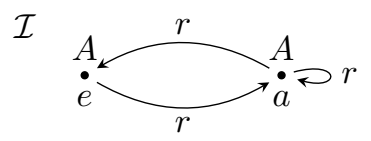

We now come to the proof of (3). We adapt the proof given in (Artale et al. 2021b) that RE existence in $\mathcal{A} \mathcal{L C O} \mathcal{O}_{u}$ is 2EXPTIME-complete. The proof is based on the bisimulation characterisation given in the previous section. The changes required to the proof in (Artale et al. 2021b) are subtle and non-trivial, however, as one now has to take care of the Condition $(\iota)$ for definite descriptions. Assume $\mathcal{O}, \Sigma$, and an individual $a$ are given. Then we say that $\mathcal{O},\{a\}$ and $\mathcal{O}, \neg\{a\}$ are jointly consistent modulo $\mathcal{A L C} \mathcal{O}_{u}^{\iota}(\Sigma)$ bisimulations iff there exist models $\mathcal{I}$ and $\mathcal{J}$ of $\mathcal{O}$ and $e \in \Delta^{\mathcal{J}}$ such that $e \neq a^{\mathcal{J}}$ and $\left(\mathcal{I}, a^{\mathcal{I}}\right) \sim_{\Sigma}^{\mathcal{A} \mathcal{L C O} \mathcal{O}_{u}^{\iota}}(\mathcal{J}, e)$. Example 2 above illustrates this definition. The following lemma reduces the $\mathrm{RE}$ existence problem to the problem of deciding the complement of joint consistency modulo $\mathcal{A L C O}_{u}^{\iota}(\Sigma)$ bisimulations.
Lemma 10. Let $\mathcal{O}$ be an $\mathcal{A} \mathcal{L C O} \mathcal{C}_{u}^{\iota}$ ontology, a an individual name, and $\Sigma$ a signature. Then the following conditions are equivalent:

1. there exists an $\mathcal{A} \mathcal{L C O}_{u}^{\iota}(\Sigma)$ RE for a under $\mathcal{O}$;

2. $\mathcal{O},\{a\}$ and $\mathcal{O}, \neg\{a\}$ are not jointly consistent modulo $\mathcal{A L C O}_{u}^{\iota}(\Sigma)$ bisimulations.

The following lemma states the main technical result from which Point (3) follows.

Lemma 11. For $\mathcal{A L C O}_{u}^{\iota}$ ontologies $\mathcal{O}$, signatures $\Sigma$, and individual names $a$, joint consistency of $\mathcal{O},\{a\}$ and $\mathcal{O}, \neg\{a\}$ modulo $\mathcal{A L C O}_{u}^{\iota}(\Sigma)$-bisimulations is 2EXPTIME-complete. The lower bound holds already if $a$ is the only individual name in $\mathcal{O}$.

Sketch. The proof of the upper bound is mosaic-based, where mosaics are pairs $\left(T_{1}, T_{2}\right)$ of sets $T_{1}$ and $T_{2}$ of $\mathcal{O}_{-}$ types such that there exist models $\mathcal{I}_{1}$ and $\mathcal{I}_{2}$ of $\mathcal{O}$ and nodes $d_{t}, t \in T_{i}$, realising $t$ in $\mathcal{I}_{i}, i=1,2$, such that all pairs $d_{t}, d_{t^{\prime}}$ with $t, t^{\prime} \in T_{1} \cup T_{2}$ are $\mathcal{A L C O}_{u}^{\iota}(\Sigma)$ bisimilar. The maximal sets of such pairs that can make up the types realised in models $\mathcal{I}_{1}, \mathcal{I}_{2}$ can be enumerated in double exponential time by formulating appropriate constraints and employing a recursive elimination procedure. These constraints have to be extended significantly compared to (Artale et al. 2021b) to deal with the cardinality constraints imposed by definite descriptions. The lower bound can be proved by adapting the lower bound proof for $\mathcal{A} \mathcal{L C O}_{u}$ in (Artale et al. 2021b).

To show Point (4), we make use of a recent undecidability proof in the context of conjunctive query inseparability of $\mathcal{A L C ~ K B s}$ given in (Botoeva et al. 2019). In that paper it is shown that it is undecidable whether two $\mathcal{A L C} \mathrm{KB}$ s entail the same conjunctive queries. To this end, an $\mathcal{A L C}$ ontology $\mathcal{O}_{1}$, an $\mathcal{E} \mathcal{L}$-ontology $\mathcal{O}_{2}$, a signature $\Sigma$ consisting of concept and role names, and a single individual name $a$ are constructed such that it is undecidable whether

$$
\mathcal{O}_{1} \cup\{r(a, a)\} \models C(a) \quad \Rightarrow \quad \mathcal{O}_{2} \cup\{r(a, a)\} \models C(a)
$$

for all $\mathcal{E} \mathcal{L}(\Sigma)$ concepts $C$. It turns out that only concepts $C$ of a particular form are relevant. In our reduction we create an $\mathcal{A L C O}$ ontology $\mathcal{O}$ by taking the union of relativized versions of $\mathcal{O}_{1}$ and $\mathcal{O}_{2}$ and two individuals $a$ and $b$ such that the relativization of $\mathcal{O}_{1}$ acts on $a$ and the relativization of $\mathcal{O}_{2}$ on $b$. We add a fresh concept name $D_{a, b}$ to $\Sigma$ and add $D_{a, b} \equiv\{a\} \sqcup\{b\}$ to $\mathcal{O}$. Then the set $\{a, b\}$ is trivially explicitly definable using an $\mathcal{E} \mathcal{L}(\Sigma)$ concept under $\mathcal{O}$ and so $a$ is explicitly definable using an $\mathcal{E} \mathcal{L} \mathcal{O}_{u}^{\iota}(\Sigma)$ concept under $\mathcal{O}$ iff it can be distinguished from $b$ using an $\mathcal{E} \mathcal{L} \mathcal{O}_{u}^{\iota}(\Sigma)$ concept in the sense that $\mathcal{O} \models C(a)$ and $\mathcal{O} \models \neg C(a)$. The undecidability of a weaker form of distinguishability (does there exist an $\mathcal{E} \mathcal{L} \mathcal{O}_{u}^{\iota}(\Sigma)$ concept such that $\mathcal{O}=C(a)$ and $\mathcal{O} \not \models C(a)$ ?) is immediate. To achieve undecidability of the stronger form of indistinguishability we add further inclusions to $\mathcal{O}$ that make subtle use of the form of the relevant concepts $C$.

We come to the proof of Point (5). It has already been observed in Point (3) of Section 2.3 that for every $\mathcal{E} \mathcal{L} \mathcal{O}_{u}^{\iota}$ ontology $\mathcal{O}, \Sigma \subseteq \Sigma_{\mathcal{O}}$, and individual name $a$ that denotes 
w.r.t. $\mathcal{O}$, there exists an $\mathcal{E} \mathcal{L} \mathcal{O}_{u}(\Sigma)$ RE for $a$ under $\mathcal{O}$ iff there exists an $\mathcal{E} \mathcal{L O}_{u}^{\iota}(\Sigma)$ RE for $a$ under $\mathcal{O}$. Thus, as we assume in Point (5) that $a$ denotes w.r.t. the ontology, it suffices to decide the existence of $\mathcal{E} \mathcal{L O}_{u}(\Sigma)$ REs. In fact, one can use Theorem 5 and the simulation characterisation of $\mathcal{E} \mathcal{L} \mathcal{O}_{u}$ concepts given in Lemma 8 to prove the following characterisation of the existence of REs.

Lemma 12. Given a signature $\Sigma$ and an $\mathcal{E} \mathcal{L} \mathcal{O}_{u}^{\iota}$ ontology $\mathcal{O}=\mathcal{O}_{0} \cup\{\mathrm{A} \equiv\{a\}\}$, where $\mathcal{O}_{0}$ is an $\mathcal{E} \mathcal{L} \mathcal{O}_{u}^{\iota}$ ontology in normal form, $\mathrm{A}$ is a concept name satisfiable w.r.t. $\mathcal{O}$, and a is an individual name, the following are equivalent:

1. there does not exist an $\mathcal{E} \mathcal{L O}_{u}(\Sigma) R E$ for a under $\mathcal{O}$;

2. there exist a model $\mathcal{J}$ of $\mathcal{O}$ and $e \in \Delta^{\mathcal{J}}$ such that $\left(\mathcal{I}_{\mathrm{A}, \mathcal{O}}, a^{\mathcal{I}_{\mathrm{A}, \mathcal{O}}}\right) \leq_{\Sigma}^{\mathcal{E} \mathcal{L} \mathcal{O}_{u}}(\mathcal{J}, e)$ and $e \neq a^{\mathcal{J}}$.

Condition (2) of Lemma 12 can be checked in polynomial time in the size of $\mathcal{O}$ (Artale et al. 2021a). Thus, to show Point (5), it suffices to recall that every $\mathcal{E} \mathcal{L} \mathcal{O}_{u}^{\iota}$ ontology can be transformed in polynomial time into a $\mathcal{E} \mathcal{L} \mathcal{O}_{u}^{\iota}$ ontology in normal form that is a conservative extension of the original ontology, and observe that the existence of an RE over a subset of the signature of an ontology is invariant under replacing the ontology by a conservative extension.

The complexity of RE existence remains open if we do not assume the individual $a$ denotes (of course, if we assume total interpretations, this is trivially the case). We note that a PTIME upper bound cannot be shown via the PBDP: while $\mathcal{E} \mathcal{L}$ enjoys the PBDP (Lutz, Seylan, and Wolter 2019), neither $\mathcal{E} \mathcal{L} \mathcal{O}^{\iota}$ nor $\mathcal{E} \mathcal{L} \mathcal{O}_{u}^{\iota}$ enjoy the PBDP for individuals on partial interpretations. To show this for $\mathcal{E} \mathcal{L} \mathcal{O}_{u}^{\iota}$ one can use an example provided in (Fortin, Konev, and Wolter 2021). The following example shows that $\mathcal{E} \mathcal{L} \mathcal{O}^{\iota}$ does not enjoy the PBDP for individuals on partial interpretations.

Example 3. Let $\Sigma=\{b, B\}$ and $\mathcal{O}$ be the $\mathcal{E} \mathcal{L} \mathcal{O}^{\iota}$ ontology:

$$
\{a\} \sqsubseteq\{b\}, \quad\{a\} \sqsubseteq \exists r . B, \quad B \sqsubseteq \exists s .\{a\} .
$$

On partial interpretations, a is implicitly definable from $\Sigma$ under $\mathcal{O}$, since $\mathcal{O} \models\{a\} \equiv\{b\} \sqcap \exists u$.B. However, $a$ is not $\mathcal{E} \mathcal{L O}^{\iota}(\Sigma)$ explicitly definable under $\mathcal{O}$, as shown by the partial interpretations $\mathcal{I}, \mathcal{J}$ that are models of $\mathcal{O}$ in the figure below, where we assume $a^{\mathcal{I}}=b$, $a^{\mathcal{J}}$ undefined, $b^{\mathcal{I}}=b$, and $b^{\mathcal{J}}=b$. Non $\mathcal{E} \mathcal{L O}^{\prime}(\Sigma)$ explicit definability follows from the observation that b satisfies exactly the same $\mathcal{E} \mathcal{L} \mathcal{O}^{\iota}(\Sigma)$ concepts in $\mathcal{I}$ and in $\mathcal{J}$ (namely $\{b\}$ ).

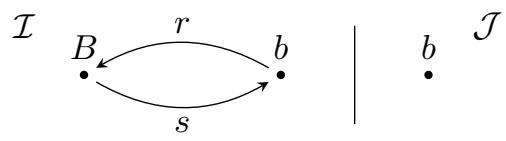

\section{Free DLs with Dual-Domain Semantics}

Free logics deal with terms that might fail to denote any existent object. Semantically, they need to address the following issues: $(i)$ the formal distinction between existent and nonexistent objects; $(i i)$ the truth of atomic formulas involving terms that do not refer to existent objects. Concerning $(i)$, the two main options are the so-called single-domain and dual-domain semantics. Single-domain semantics are based on interpretations with a unique domain of objects, representing the set of things that possibly exist. Dual-domain semantics introduce instead interpretations based on two domains of objects: the outer domain, representing the set of all possible things; and the inner domain, a subset of the outer domain over which quantifiers are allowed to range, containing only those objects that actually exist. Concerning (ii), three of the most prominent options are the so-called positive, negative and neutral (or gapped) semantics. Positive semantics allow atomic formulas with terms that refer to non-existent objects to be possibly true. Negative semantics require all atomic formulas with terms denoting non-existent objects, or non-denoting at all, to be false by default. Neutral semantics introduce a truth-value gap for such formulas, so that their truth value is left undefined (Nolt 2020).

While partial interpretations are a kind of single-domain negative semantics, Neuhaus, Kutz, and Righetti (2020) consider free DLs with nominals over dual-domain interpretations, presenting a positive, a negative and a gapped semantics. This approach naturally fits scenarios where objects of the domain can start or cease to exist, as frequently considered in first-order modal and temporal logics (Garson 2001; Gabbay et al. 2003; Braüner and Ghilardi 2007; Fitting and Mendelsohn 2012; Garson 2013). Moreover, a positive semantics allows one to avoid inconsistencies when reasoning in presence of data sources that contradict the ontology, a motivation shared also by inconsistency-tolerant DLs (Lembo et al. 2010; Lembo et al. 2015), by representing "error" individuals as non-existent objects.

To show how the dual-domain semantics can be captured in our framework, we present the logic $\mathcal{A L C} \mathcal{L}^{\iota *}$ (Neuhaus, Kutz, and Righetti 2020), defined similarly to $\mathcal{A} \mathcal{L C} \mathcal{O}^{\iota}$, with the addition of $\mathbf{T}$ as a concept name representing the set of existing objects. Moreover, an $\mathcal{A L C O}^{\iota *}$ assertion is of the form $C(\tau), r\left(\tau_{1}, \tau_{2}\right)$, or $\tau_{1}=\tau_{2}$, with $C$ an $\mathcal{A L C O}^{\iota *}$ concept, $r \in \mathrm{N}_{\mathrm{R}}$, and $\tau, \tau_{1}, \tau_{2} \mathcal{A} \mathcal{L} \mathcal{C O}{ }^{\iota *}$ terms. An $\mathcal{A L C O}^{\iota *}$ axiom is either an $\mathcal{A} \mathcal{L C} \mathcal{O}^{\iota *} \mathrm{CI}, C \sqsubseteq D$, with $C, D \mathcal{A L C O}{ }^{\iota *}$ concepts, or an $\mathcal{A} \mathcal{L C O} \mathcal{O}^{\iota *}$ assertion. An $\mathcal{A L C O}{ }^{\iota *}$ formula is defined inductively as follows, with $\beta \mathcal{A L C O}{ }^{\iota *}$ axiom:

$$
\varphi::=\beta|\neg(\varphi)|(\varphi \wedge \varphi) \text {. }
$$

A dual-domain interpretation is a triple $I=\left(\Delta^{I}, \mathfrak{d}^{I}, \cdot^{I}\right)$, where $\Delta^{I}$ is a non-empty set, called outer domain of $I, \mathfrak{d}^{I} \subset$ $\Delta^{I}$ is a (possibly empty) set called inner domain of $I$, and ${ }^{I}$ is a function mapping every $A \in \mathrm{N}_{\mathrm{C}}$ to a subset of $\Delta^{I}$, every $r \in \mathrm{N}_{\mathrm{R}}$ to a subset of $\Delta^{I} \times \Delta^{I}$, and every $a \in \mathrm{N}_{\mathrm{I}}$ to an element in $\Delta^{I}$. Given a dual-domain interpretation $I$, the extension $C^{I}$ of an $\mathcal{A L C O}{ }^{\text {i* }}$ concept $C$ in $I$ is defined similarly to $\mathcal{A} \mathcal{L C O} \mathcal{O}^{\iota}$, with the exception of:

$$
\begin{gathered}
\mathbf{T}^{I}=\mathfrak{d}^{I}, \\
(\exists r . C)^{I}=\left\{d \in \Delta^{I} \mid \exists e \in \mathfrak{d}^{I} \cap C^{I}:(d, e) \in r^{I}\right\},
\end{gathered}
$$

while, for a definite description $\iota C$, the value of $\iota C$ in $I$ is:

$$
(\iota C)^{I}= \begin{cases}d, & \text { if } \mathfrak{d}^{I} \cap C^{I}=\{d\} \\ d_{\iota C}, & \text { with } d_{\iota C} \in \Delta^{I} \backslash \mathfrak{d}^{I} \text { arbitrary, otherwise. }\end{cases}
$$

Moreover, on dual-domain interpretations, we define the extension of $\mathcal{A} \mathcal{L C O} \mathcal{O}^{\iota *}$ nominals as follows: $\{\tau\}^{I}=\left\{\tau^{I}\right\}$. 
Given an $\mathcal{A} \mathcal{L C O}^{\iota *}$ formula $\varphi$ and a dual-domain interpretation $I$, we inductively define two different kinds of satisfaction relations between $I$ and $\varphi$ : one under positive semantics, denoted by $\models^{+}$; and one under negative semantics, denoted by $\mid=^{-}$. In the following, $\circ \in\{+,-\}$.

$$
\begin{gathered}
I \models{ }^{\circ} C(\tau) \text { iff } \begin{cases}\tau^{I} \in C^{I}, & \text { if } \circ=+; \\
\tau^{I} \in \mathfrak{d}^{I} \text { and } \tau^{I} \in C^{I}, & \text { if } \circ=-;\end{cases} \\
I \models{ }^{\circ} r\left(\tau_{1}, \tau_{2}\right) \text { iff } \begin{cases}\left(\tau_{1}^{I}, \tau_{2}^{I}\right) \in r^{I}, & \text { if } \circ=+; \\
\tau_{1}^{I}, \tau_{2}^{I} \in \mathfrak{d}^{I} \text { and }\left(\tau_{1}^{I}, \tau_{2}^{I}\right) \in r^{I}, & \text { if } \circ=-;\end{cases} \\
I \models{ }^{\circ} \tau_{1}=\tau_{2} \text { iff } \begin{cases}\tau_{1}^{I}=\tau_{2}^{I}, & \text { if } \circ=+; \\
\tau_{1}^{I}, \tau_{2}^{I} \in \mathfrak{d}^{I} \text { and } \tau_{1}^{I}=\tau_{2}^{I}, & \text { if } \circ=-;\end{cases} \\
I=^{\circ} C \sqsubseteq D \text { iff, for all } d \in \mathfrak{d}^{I}, d \in C^{I} \text { implies } d \in D^{I},
\end{gathered}
$$

We say that $\varphi$ is satisfiable on dual-domain interpretations under positive, respectively negative, semantics iff there exists a dual-domain interpretation $I$ such that $I==^{+} \varphi$, respectively $I \models=^{-} \varphi$.

Differently from classical $\mathcal{A L C O}$ on total interpretations and from $\mathcal{A L C O}_{u}^{\iota}$ on partial interpretations (cf. Point (2) in Section 2.3), $\mathcal{A} \mathcal{L C} \mathcal{O}^{\iota *}$ assertions under these semantics cannot be encoded into $\mathcal{A} \mathcal{L C O}^{\iota *}$ CIs. Moreover, parentheses in negated formulas $\neg(\varphi)$ are not eliminable, since they disambiguate between assertions of the form $\neg C(\tau)$, with a negated concept, and negated assertions of the form $\neg(C(\tau))$. Indeed, these expressions have different satisfaction conditions on dual-domain interpretations under negative semantics: while $\neg C(\tau)$ requires $\tau^{I}$ to be an element of the inner domain in any of its models $I$, a formula like $\neg(C(\tau))$ is satisfied also in dual-domain interpretations $I$ where $\tau^{I}$ is in the outer, but not in the inner, domain.

We now show that $\mathcal{A} \mathcal{L C O}^{\iota *}$ satisfiability on dual-domain interpretations under either positive or negative semantics is polynomial time reducible to $\mathcal{A} \mathcal{L C O} \mathcal{O}_{u}^{\iota}$ reasoning on partial interpretations. The proof is reminiscent of the one from first-order modal logic, to reduce varying to constant domain semantics (Braüner and Ghilardi 2007; Fitting and Mendelsohn 2012), in that it exploits an existence concept to represent the inner domain on partial interpretations.

Theorem 13. $\mathcal{A L C O}^{\iota *}$ formula satisfiability on dual domain interpretations under either positive or negative semantics is polynomial time reducible to $\mathcal{A L C O}_{u}^{\iota}$ ontology satisfiability.

Since the gapped semantics allows for truth value gaps, it is not covered by our setting based on a two-valued semantics. A comparison with this option is left as future work.

\section{Related Work}

Definite descriptions introduce mild forms of cardinality constraints, a set of constructors with a long tradition in DLs (Baader, Buchheit, and Hollunder 1996; Tobies 2000; Baader and Ecke 2017; Baader and Bortoli 2019; Baader, Bednarczyk, and Rudolph 2020) that allow to constrain the number of elements in the extension of a concept. The expressivity of many of these logics goes far beyond the DLs proposed here, and novel reasoning tools are required. In contrast, reasoning in our free DLs can be reduced to reasoning in standard DLs $\left(\mathcal{A L C O} \mathcal{O}_{u}^{\iota}\right)$ or mild extensions $\left(\mathcal{E} \mathcal{L} \mathcal{O}_{u}^{\iota}\right)$.

Concerning RE generation tasks, other DL-based approaches have studied the problem of finding a concept to describe an element with respect to a single interpretation given as input (Areces, Koller, and Striegnitz 2008; Areces, Figueira, and Gorín 2011). More expressive DLs, as well as a relaxed version of the closed-world assumption, are considered in (Ren, van Deemter, and Pan 2010b; Ren, van Deemter, and Pan 2010a).

REs have also been proposed for several applications in ontology-based data management, such as query answering over KBs (Borgida, Toman, and Weddell 2016b; Borgida, Toman, and Weddell 2017; Toman and Weddell 2019a), identity resolution in ontology-based data access (Toman and Weddell 2018; Toman and Weddell 2019b), and identification problems in conceptual modelling (Borgida, Toman, and Weddell 2016a). The DLs considered in these papers are tractable languages tailored to efficient query answering in presence of functionality and path-based identification constraints. In this approach, DL concepts can serve as REs under a given $\mathrm{KB}$ if they contains exactly one element in all the models of the KB and satisfy a correctness condition with respect to a query. They are not, however, directly treated as possibly non-denoting terms of the language.

Finally, hybrid logics with non-denoting nominals have not received much attention in the literature, with the exception of (Hansen 2011) in the context of public announcement logics. However, formalisms involving definite descriptions are actively investigated in first-order modal logic (Indrzejczak 2018; Orlandelli and Corsi 2018; Indrzejczak 2020), where the possible lack of referents for names and descriptions is usually paired with non-rigid denotation features, i.e., the ability to refer to different objects at different states.

\section{Discussion}

We have introduced DLs with definite descriptions on partial interpretations, and investigated standard reasoning (satisfiability and entailment) and automated support for generating definite descriptions (RE existence). Many open problems remain to be explored. Regarding $\mathcal{E} \mathcal{L} \mathcal{O}_{u}^{\iota}$, it is open whether the PTIME upper bound for RE existence holds in general, whether there is a polynomial size canonical model for $\mathcal{E} \mathcal{L} \mathcal{O}_{u}^{\iota}$-concepts and whether a satisfactory model-theoretic characterisation of its expressivity can be given. Also, RE existence is only a first step towards automated support for generating definite descriptions in practice. This could be approached by exploring the shape and interpretability of definitions obtained from interpolants computed by FO theorem provers. Finally, we intend to extend our free DLs with definite descriptions with a temporal dimension (Lutz, Wolter, and Zakharyaschev 2008), for applications in temporal conceptual modelling and query answering over temporal DL ontologies (Artale et al. 2014; Artale et al. 2017), where the interaction between lack of denotation and non-rigidity can be at stake. 


\section{Acknowledgements}

Ozaki was supported by the Norwegian Research Council, project number 316022. Wolter was supported by EPSRC EP/S032207/1.

\section{References}

Areces, C.; Blackburn, P.; and Marx, M. 2001. Hybrid logics: Characterization, interpolation and complexity. $J$. Symb. Log. 66(3):977-1010.

Areces, C.; Figueira, S.; and Gorín, D. 2011. Using logic in the generation of referring expressions. In $L A C L, 17-32$.

Areces, C.; Koller, A.; and Striegnitz, K. 2008. Referring expressions as formulas of description logic. In INLG.

Artale, A.; Kontchakov, R.; Ryzhikov, V.; and Zakharyaschev, M. 2014. A cookbook for temporal conceptual data modelling with description logics. ACM Trans. Comput. Log. 15(3):25:1-25:50.

Artale, A.; Kontchakov, R.; Kovtunova, A.; Ryzhikov, V.; Wolter, F.; and Zakharyaschev, M. 2017. Ontologymediated query answering over temporal data: A survey (invited talk). In TIME, 1:1-1:37.

Artale, A.; ; Mazzullo, A.; Ozaki, A.; and Wolter, F. 2021a. On free description logics with definite descriptions. Available at https://arxiv.org/abs/2106.15513.

Artale, A.; Jung, J. C.; Mazzullo, A.; Ozaki, A.; and Wolter, F. 2021b. Living without Beth and Craig: Definitions and interpolants in description logics with nominals and role inclusions. In AAAI, 6193-6201.

Baader, F., and Bortoli, F. D. 2019. On the expressive power of description logics with cardinality constraints on finite and infinite sets. In FroCoS, 203-219.

Baader, F., and Ecke, A. 2017. Extending the description logic $\mathcal{A L C}$ with more expressive cardinality constraints on concepts. In GCAI, 6-19.

Baader, F.; Bednarczyk, B.; and Rudolph, S. 2020. Satisfiability and query answering in description logics with global and local cardinality constraints. In ECAI, 616-623.

Baader, F.; Calvanese, D.; McGuinness, D. L.; Nardi, D.; and Patel-Schneider, P. F., eds. 2003. The Description Logic Handbook: Theory, Implementation, and Applications. Cambridge University Press.

Baader, F.; Brandt, S.; and Lutz, C. 2005. Pushing the $\mathcal{E} \mathcal{L}$ envelope. In IJCAI, 364-369.

Baader, F.; Buchheit, M.; and Hollunder, B. 1996. Cardinality restrictions on concepts. Artif. Intell. 88(1-2):195-213.

Bencivenga, E. 2002. Free logics. In Handbook of Philosophical Logic. Springer. 147-196.

Beth, E. W. 1956. On Padoa's Method in the Theory of Definition. Journal of Symbolic Logic 21(2):194-195.

Borgida, A.; Toman, D.; and Weddell, G. E. 2016a. On referring expressions in information systems derived from conceptual modelling. In ER, 183-197.

Borgida, A.; Toman, D.; and Weddell, G. E. 2016b. On referring expressions in query answering over first order knowledge bases. In $K R, 319-328$.
Borgida, A.; Toman, D.; and Weddell, G. E. 2017. Concerning referring expressions in query answers. In IJCAI, 4791-4795.

Botoeva, E.; Lutz, C.; Ryzhikov, V.; Wolter, F.; and Zakharyaschev, M. 2019. Query inseparability for $\mathcal{A L C}$ ontologies. Artif. Intell. 272:1-51.

Braüner, T., and Ghilardi, S. 2007. First-order Modal Logic. In Handbook of Modal Logic. Elsevier. 549-620.

Cann, R. 1993. Formal Semantics: an Introduction. Cambridge University Press.

Chang, C. C., and Keisler, H. J. 1990. Model Theory. Elsevier.

Fitting, M., and Mendelsohn, R. L. 2012. First-order Modal Logic. Springer Science \& Business Media.

Fortin, M.; Konev, B.; and Wolter, F. 2021. Interpolants and Explicit Definitions in Horn Description Logics. Submitted to Description Logic Workshop.

Gabbay, D. M.; Kurucz, A.; Wolter, F.; and Zakharyaschev, M. 2003. Many-dimensional Modal Logics: Theory and Applications. North Holland Publishing Company.

Garson, J. W. 2001. Quantification in modal logic. In Handbook of philosophical logic. Springer. 267-323.

Garson, J. W. 2013. Modal logic for Philosophers. Cambridge University Press.

Hansen, J. U. 2011. A hybrid public announcement logic with distributed knowledge. Electr. Notes Theor. Comput. Sci. 273:33-50.

Indrzejczak, A. 2018. Cut-free modal theory of definite descriptions. In AiML, 387-406.

Indrzejczak, A. 2020. Existence, definedness and definite descriptions in hybrid modal logic. In 13th Conference on Advances in Modal Logic, AiML 2020, Helsinki, Finland, August 24-28, 2020, 349-368.

Krahmer, E., and van Deemter, K. 2012. Computational generation of referring expressions: A survey. Computational Linguistics 38(1):173-218.

Krahmer, E.; van Erk, S.; and Verleg, A. 2003. Graphbased generation of referring expressions. Computational Linguistics 29(1):53-72.

Lehmann, S. 2002. More free logic. In Handbook of Philosophical Logic. Springer. 197-259.

Lembo, D.; Lenzerini, M.; Rosati, R.; Ruzzi, M.; and Savo, D. F. 2010. Inconsistency-tolerant semantics for description logics. In $R R, 103-117$.

Lembo, D.; Lenzerini, M.; Rosati, R.; Ruzzi, M.; and Savo, D. F. 2015. Inconsistency-tolerant query answering in ontology-based data access. J. Web Semant. 33:3-29.

Lutz, C., and Wolter, F. 2010. Deciding inseparability and conservative extensions in the description logic EL. J. Symb. Comput. 45(2):194-228.

Lutz, C.; Piro, R.; and Wolter, F. 2011. Description Logic TBoxes: Model-Theoretic Characterizations and Rewritability. In IJCAI, 983-988. 
Lutz, C.; Seylan, I.; and Wolter, F. 2019. The data complexity of ontology-mediated queries with closed predicates. Log. Methods Comput. Sci. 15(3).

Lutz, C.; Wolter, F.; and Zakharyaschev, M. 2008. Temporal description logics: A survey. In TIME, 3-14.

Neale, S. 1990. Descriptions. MIT Press.

Neuhaus, F.; Kutz, O.; and Righetti, G. 2020. Free description logic for ontologists. In $B O S K$.

Nolt, J. 2020. Free Logic. In Zalta, E. N., ed., The Stanford Encyclopedia of Philosophy. Metaphysics Research Lab, Stanford University, winter 2020 edition.

Orlandelli, E., and Corsi, G. 2018. Labelled Calculi for Quantified Modal Logics with Non-rigid and Non-denoting Terms. In ARQNL@IJCAR, 64-78.

Passy, S., and Tinchev, T. 1991. An essay in combinatory dynamic logic. Inf. Comput. 93(2):263-332.

Reiter, E., and Dale, R., eds. 2000. Building Natural Language Generation Systems. Cambridge University Press.

Ren, Y.; van Deemter, K.; and Pan, J. Z. 2010a. Charting the potential of description logic for the generation of referring expressions. In INLG.

Ren, Y.; van Deemter, K.; and Pan, J. Z. 2010b. Generating referring expressions with OWL2. In $D L$.

Russell, B. 1905. On Denoting. Mind 14(56):479-493.

ten Cate, B.; Conradie, W.; Marx, M.; and Venema, Y. 2006. Definitorially complete description logics. In $K R, 79-89$.

ten Cate, B.; Franconi, E.; and Seylan, I. 2013. Beth definability in expressive description logics. J. Artif. Intell. Res. 48:347-414.

ten Cate, B. 2005. Model theory for Extended Modal Languages. Ph.D. Dissertation, University of Amsterdam. ILLC Dissertation Series DS-2005-01.

Tobies, S. 2000. The complexity of reasoning with cardinality restrictions and nominals in expressive description logics. J. Artif. Intell. Res. 12:199-217.

Toman, D., and Weddell, G. E. 2018. Identity resolution in conjunctive querying over DL-based knowledge bases. In $D L$.

Toman, D., and Weddell, G. E. 2019a. Finding ALL answers to OBDA queries using referring expressions. In $A I, 117-$ 129.

Toman, D., and Weddell, G. E. 2019b. Identity resolution in ontology based data access to structured data sources. In PRICAI Part I, 473-485. 